Thorax (1951), 6, 56.

\title{
HIATAL HERNIA AND SHORT OESOPHAGUS IN CHILDREN
}

BY

\author{
ERIK HUSFELDT, GREGERS THOMSEN, AND ERIK WAMBERG \\ From the Departments of Thoracic Surgery, Radiology, and Paediatrics, the University \\ Hospital, Copenhagen
}

(RECEIVED FOR PUBLICATION SEPTEMBER 26, 1950)

Diseases and malformations of the oesophagus have hitherto been a neglected field in paediatrics. During recent years this attitude has been altered, because the progress of thoracic surgery has made operations on the oesophagus a much safer procedure and has thus increased the possibility of treating surgically oesophageal lesions in children. Among the diseases and malformations which have become the object of renewed interest is congenital hiatal hernia coexisting with a shortening of the oesophagus, which Scandinavian and French workers are accustomed to call "brachyoesophagus."

\section{Previous RePORTS}

A review of about 70 cases reported up to 1947 can be found in a paper published by one of us (Thomsen, 1949). In addition to several single cases, two large series were published in 1948, one from England and one from America, Allison reporting seven cases and Olsen and Harrington eight among children. Considering that the condition has been known for almost 30 years, the number of published cases, about 100 , is not impressive, and, judging by the literature, short oesophagus would appear to be rare among children. It would seem, however, that the literature does not convey the proper impression, since we have been able to collect 24 cases diagnosed within the last decade, 14 of them since 1947.

\section{AETIOLOGY}

As stated in the previous paper (Wamberg, 1947) some disagreement has existed as to the aetiology. According to Bund a persistence of the right pneumato-enteric recess produces a hernia in which the stomach forms the hernial contents. Tondorff, on the other hand, claims that the caudal shift of the diaphragm and abdominal contents, which normally takes place in the fourth to fifth foetal week, is disturbed so that the longitudinal growth of the oesophagus ceases and a major or minor portion of the stomach remains in the thoracic cavity, whereas the diaphragm, completing its caudal movement, envelops the thoracic stomach in a thin layer of connective tissue. Recent papers, particularly from England (Allison, 1948), have shown that none of these theories can be accepted. The fact that short oesophagus and peptic ulcer of the oesophagus are met with in a comparatively large number of subjects over 50 years of age with no history of dyspeptic symptoms militates against the idea of a congenital defect. In 1926 Akerlund described a form of hiatal hernia, occurring particularly among elderly individuals, in which the hernial 
.contents were the cardia and the adjacent part of the fundus of the stomach, the oesophagus being of normal length. This variety of hernia was usually visualized only in the horizontal position. It has now been shown by Allison that in these patients there is an incompetence of the closing mechanism of the cardial sphincter. In the horizontal position there will be a reflux of gastric contents into the hernia, whence they proceed into the oesophagus, since the resistance offered by the cardial sphincter is minimal. If the production of acid is preserved, the susceptible oesophageal mucosa will suffer and the result will be oesophagitis, possibly followed later by peptic ulcer. After some time such oesophagitis, with or without ulceration, may lead to cicatricial changes with shortening and fixing of the oesophagus.

This explanation of the aetiology of short oesophagus in adults does not, however, apply directly to children. Although short oesophagus coexists with peptic ulcer in a rather large proportion of the children due to regurgitation of acid gastric contents, the short oesophagus might easily be the primary feature. This is not, however, indicated by the operative results in our series, which seem to suggest that in children as well as in adults hiațal hernia is primary. In children the oesophagus possesses considerable powers of spontaneous retraction, giving it the appearance of being too short; later this shortening may become fixed as a peptic ulcer develops.

In addition to the four cases already published from the Queen Louise Hospital for Children in 1947 and one case from the Sundby Hospital in Copenhagen, we have succeeded in collecting 19 cases of short oesophagus from the University Hospital in Copenhagen. The age and sex distribution will be seen in the table. As far as the older patients are concerned, the diagnoses were proved by perusal of the case reports and revision of the old radiographs. Of these 24 patients, 10 were treated by surgical and 14 by conservative measures. The table sets out the present age of the patients, the time of onset of the disease, age upon first admission, number of admissions in the course of time, and, lastly, the most outstanding symptoms. A perusal of the case histories leaves the following impression of the course and symptoms of the disease.

\section{SYMPTOMS}

As a rule the symptoms manifest themselves immediately after birth or in the course of the first month of life, rarely later and, if so, usually when the child begins to take solid food. The most outstanding symptom is vomiting, which occurred in $100 \%$ of our series: it is nearly always periodical with intervening remissions of a few weeks up to several months. During the attacks the child suffers from daily vomiting or spitting, usually during or shortly after meals. The vomitus is brought up without difficulty. (In one of the children it welled out of the mouth when he stood on his head.) The amount varies within wide limits and the vomitus is usually alimentary, slightly slimy, and not infrequently blood-tinged.

The symptom next in order of frequency is haemorrhage (in $70 \%$ of cases) in the form of a slight bloody admixture in the vomitus or of regular haematemesis of extremely varying intensity. The latter is nearly always caused by the coexistent oesophagitis or a complicating peptic ulcer of the oesophagus. The haemorrhages may be alarming, and simple anaemia is no uncommon finding. Blood transfusions may be required. 


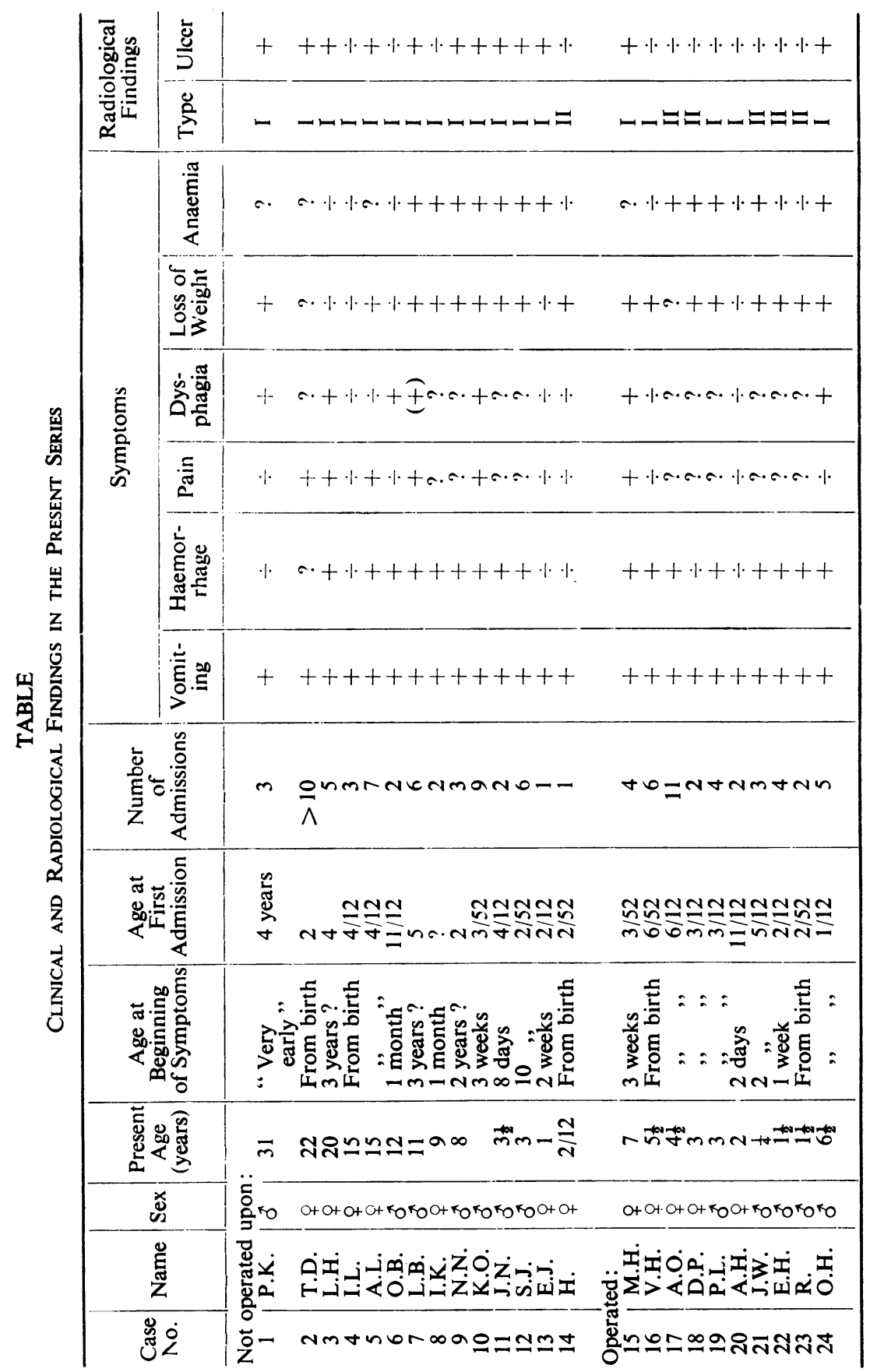


In addition to these symptoms, older children may complain of dysphagia and pain occurring in varying intensity during meals and localized at the lower part of the sternum or high in the epigastrium. It is a characteristic feature in numerous cases that the pain is most marked in the horizontal position, subsiding or disappearing altogether when the child is sitting. (One of our patients had formed the habit of sleeping in the sitting position.) In addition to the pain, a few older children may exhibit regular dysphagia, mostly for solids with marked periodicity, no doubt due to exacerbation of the complicating oesophagitis and/or oesophageal ulcer. These patients, who have a feeling that the food is being held up in the oesophagus, are able to take solid food only in small portions which have to be thoroughly masticated and swallowed with large amounts of fluid.

Such recurrent vomiting and pain, which do not infrequently make the child refuse to eat, often result in considerable loss of weight, perhaps accompanied by a disturbance of the water balance and a poor general condition. The periodicity of the symptoms is an outstanding feature of the condition, and the patients have usually been in hospital several times. The symptoms and the periodicity of the disease are illustrated by the following typical case history.

\section{CASE REPORT}

M. H., a girl, was born on July 29, 1942. (University Hospital case record 890/47.) The vomiting set in at the age of 2 to 3 weeks. It was not profuse, but was at times explosive, and the vomitus was often blood-tinged. The condition remained unchanged during the first year of life, after which it grew more periodical, as remissions of up to a fortnight would intervene. At the age of 5 the condition grew much worse. The patient developed daily vomiting, usually immediately after or exceptionally a few hours after meals. She failed to thrive, gradually lost weight, and suffered from dysphagia and sometimes from a feeling of being suffocated at meals. She was only able to take fluids. She had been in hospital four times, at 3 weeks, 8 months, 4 years, and 5 years of age. The first three times the treatment had been dietetic and symptomatic, but after the last admission she was transferred to the Ear, Nose, and Throat Department, where she obtained considerable relief from bouginage.

In April, 1949, the vomiting increased and the patient had a feeling that the food was being held up behind the sternum. Bouginage was resumed. In June, 1949, transthoracic herniotomy was performed in the Department of Thoracic Surgery on the diagnosis of hiatal hernia of the oesophagus with oesophageal ulcer.

Radiological examination of the oesophagus and stomach in 1947 had revealed marked dilatation of the upper end of the oesophagus. On a level with the eighth to ninth dorsal vertebrae the opaque medium was held up (Fig. 1a), and the oesophagus did not start emptying until somewhat later. Examination a fortnight later showed a less marked dilatation and the opaque meal passed more easily down: below the dilatation there was a constriction and the oesophagus opened directly into a hiatal hernia (Fig. 1b) about the size of a walnut. A fortnight later the dilatation had subsided almost completely and an ulcer niche, as large as a hempseed (Fig. 1c), was observed at the site of the constriction. A diagnosis of short oesophagus and oesophageal ulcer was made on the basis of these findings.

Radiological examination in 1949 showed the same changes.

Radiological control after the operation showed that the hernia had been reduced, but there was still an oesophageal constriction at the site of the ulceration (Fig. 1d). 


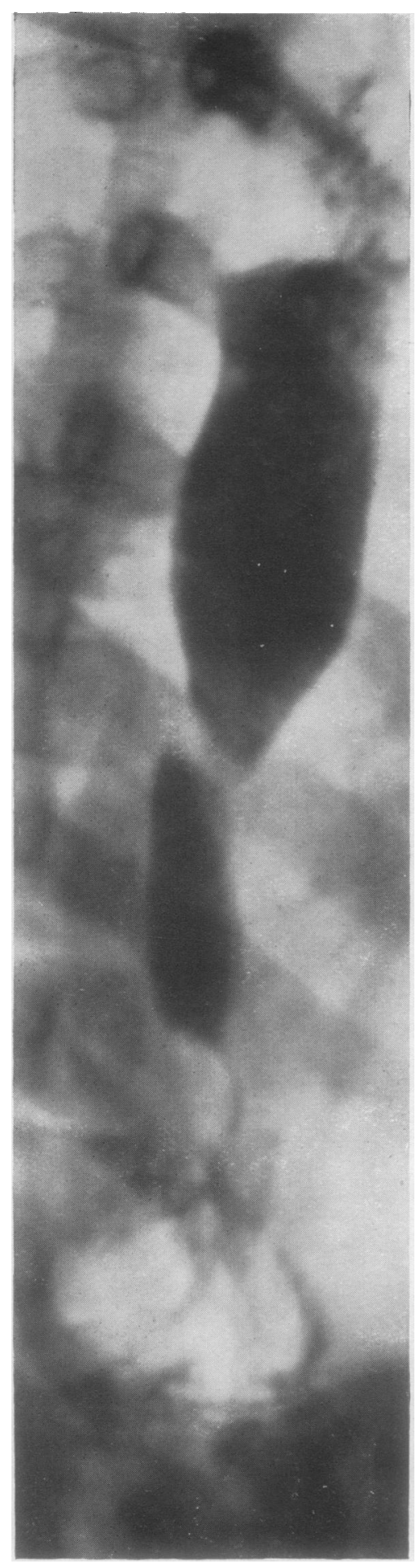

FIG. $1 b$

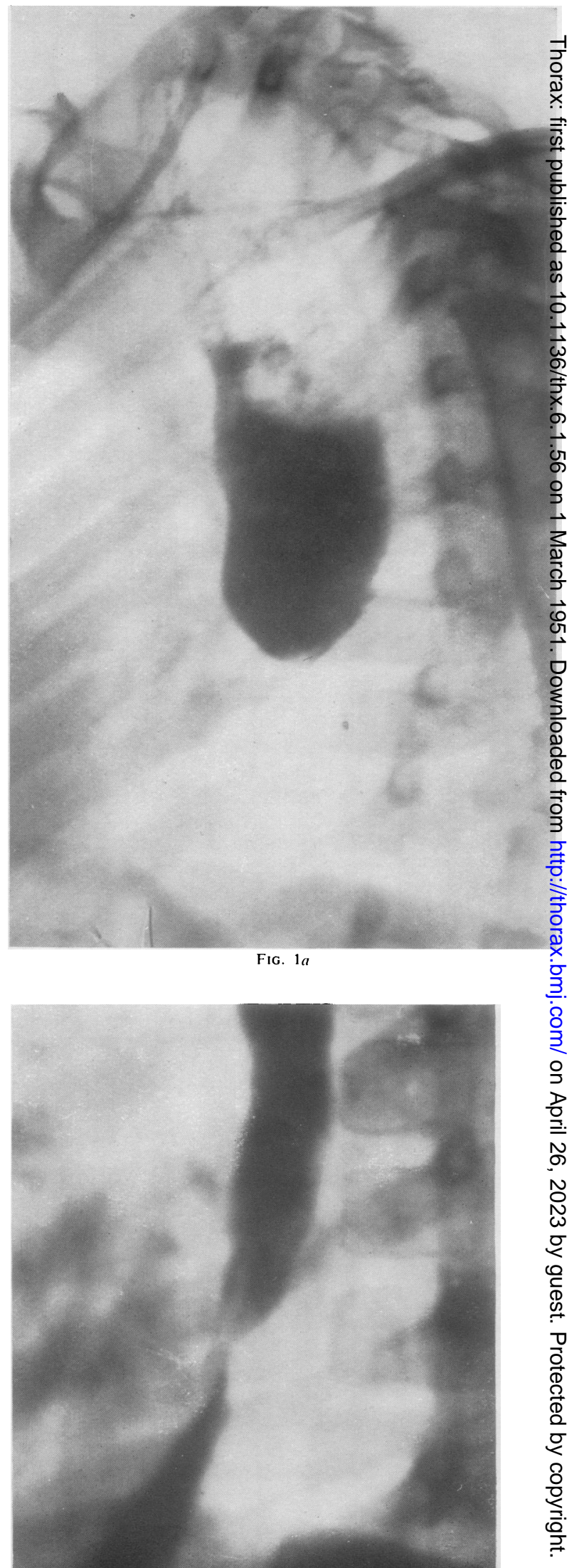

Fro. 1c 
Fig. 1a.-Marked dilatation of the upper end of the oesophagus.

FIG. 1b.-Radiograph showing oesophagus opening directly into a hiatal hernia.

Fig. 1c.-Radiograph showing subsidence of the dilatation and a marked ulcer niche.

Fig. 1d.-Hernia controlled after operation, but oesophageal constriction still present at the site of ulceration.

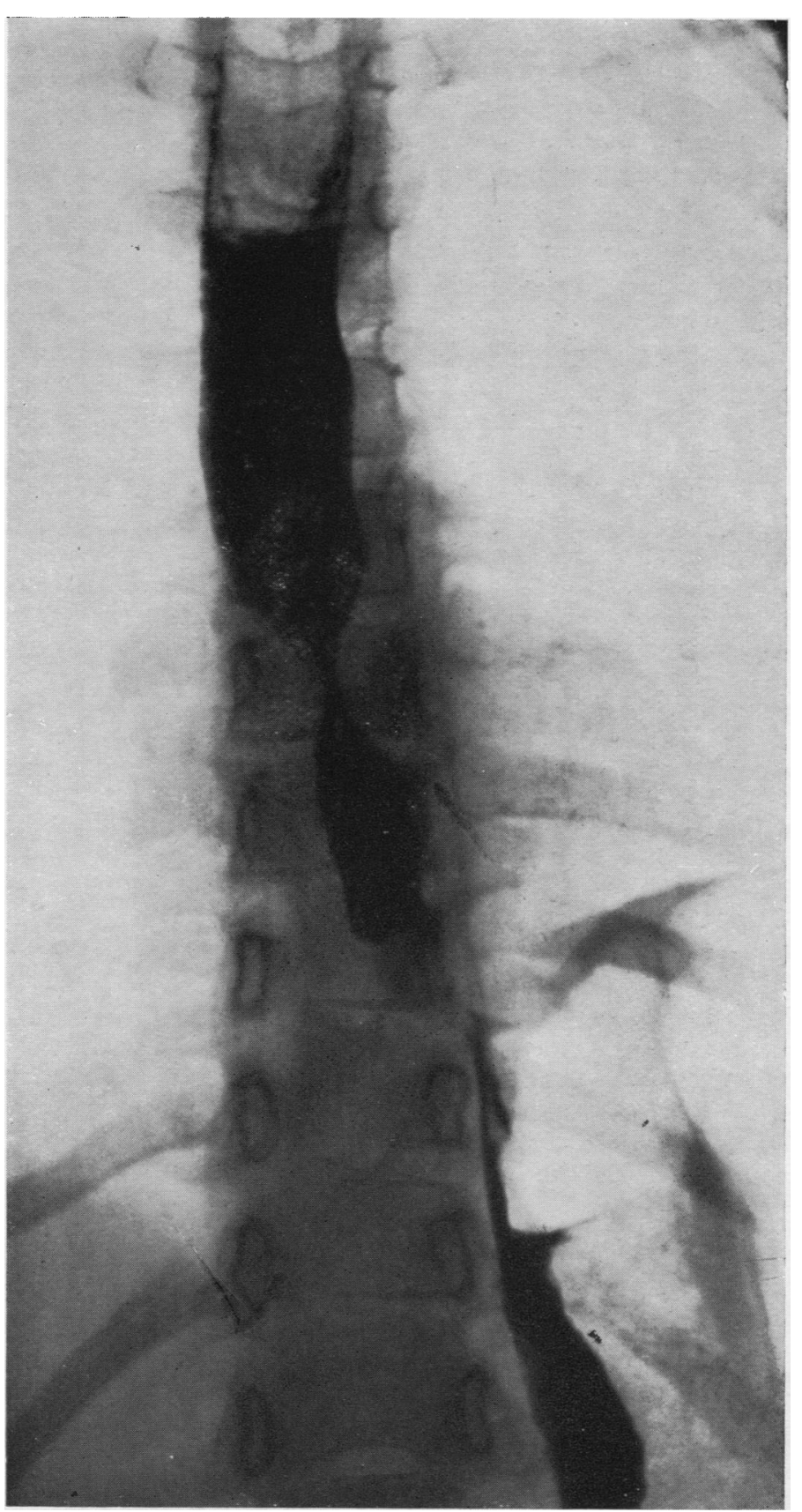

Fio. 1d 


\section{DiAgnosis}

In a few cases a diagnosis of short oesophagus can be made on the past history and the objective findings, but in the majority of cases radiological examination is required. Moreover, the clinical diagnosis must always be confirmed by radiology. On the basis of our series, we feel justified in distinguishing two types of short oesophagus, but the difference is apparent only; we presume the first variety to be a further development of the second and to be due to secondary changes (Fig. 2).

First Type of Short Oesophagus.-The first variety, shown in 18 patients, is characterized by the following changes. The oesophagus is shorter than normal, sometimes of normal width, but usually somewhat dilated. It opens directly into the hiatal hernia. The latter may be of varying size, and in some instances a rather large portion of the fundus of the stomach is above the diaphragm. At the lower end of the oesophagus, at the site of its opening into the hernia, there is a constriction indicating the cardia. This site is usually on a level with the seventh to eighth dorsal vertebrae. The oesophagus

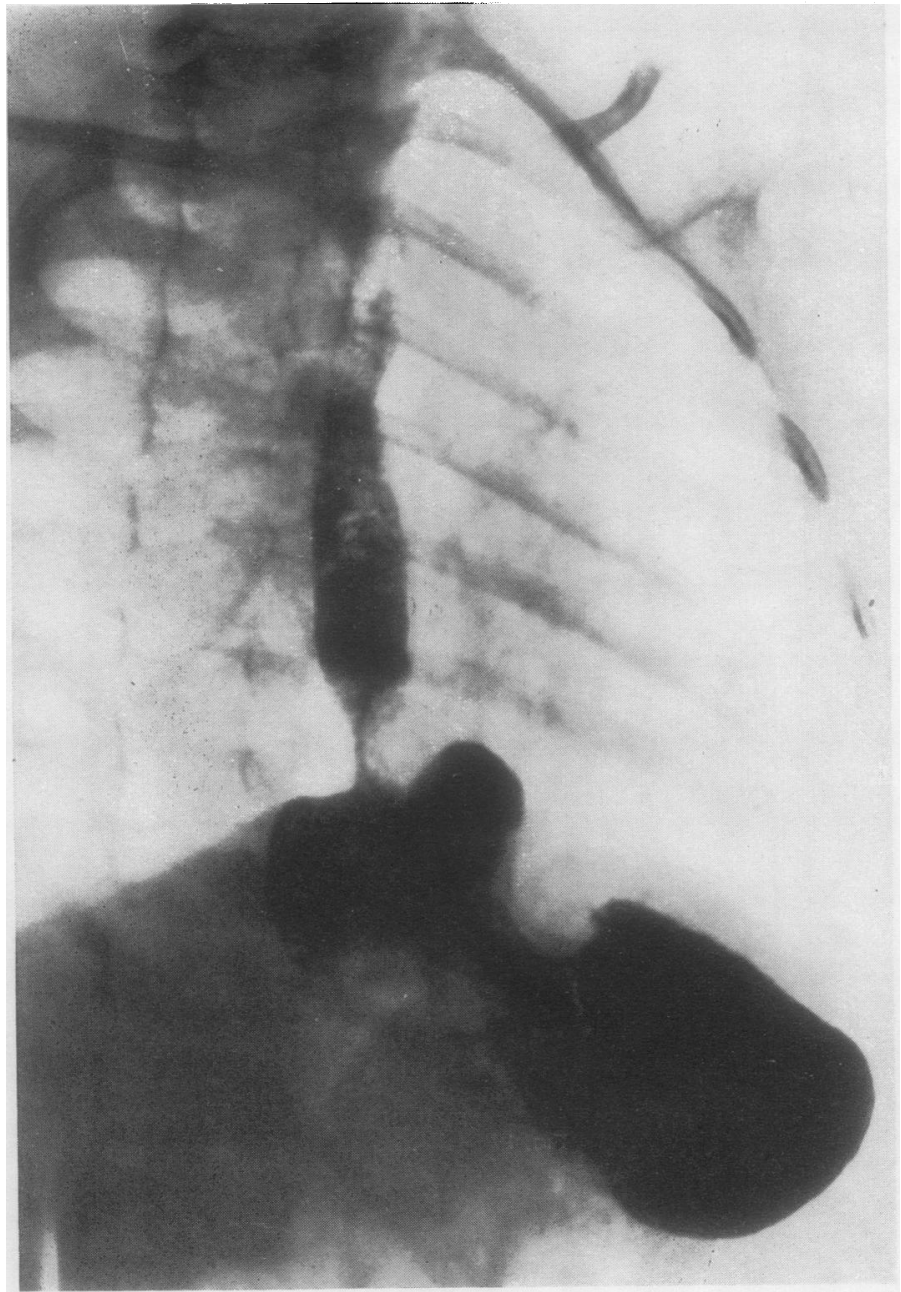
may sometimes show rather few longitudinal mucosal folds, but often there is no mucosal pattern. The hernia, on the other hand, always contains more numerous regular, or more often irregular, mucosal folds, and it is possible to point out a continuation of the relief of the gastric mucosa through the hiatus oesophagus into the hernia. The part of the stomach passing through the hiatus is nearly always broad, showing that the hiatus is larger than normal. Consequently, the upper part of the abdominal stomach converges towards the hiatus, acquiring a funnel shape unlike the normal fundus, the upper contour of which is rounded due to the acute angle formed where the oesophagus opens into the stomach high on the lesser curvature.

In all our cases, regurgitation of the barium meal from the stomach into the hernia and further into the oesophagus was demonstrable by fluoroscopy. To this sign, showing that the closing mechanism of the cardia is incompetent, we attach the utmost

Fio. 2. 
diagnostic significance. Massive reflux into the oesophagus is presumably pathognomonic of short oesophagus. So far, it has not been possible to find this sign in normal children. A sharp distinction must be made between regurgitation, which is a passive alteration of the distribution of opaque medium, due partly to the incompetent cardia, partly to external actions, and vomiting caused by gastric contractions. In a few cases the reflux of contrast medium into the oesophagus may elicit vomiting. In some children, regurgitation occurs as soon as they lie down, but at other times not until they lie prone. Screening may show how the filling of the oesophagus varies synchronously with the respiration due to alterations in the intrathoracic pressure. Similarly, an increase in the intra-abdominal pressure caused by compression of the abdomen may result in increased flow into the oesophagus. Other children have to be placed in Trendelenburg's position to fill the hernia as completely as possible. By tilting the table, the

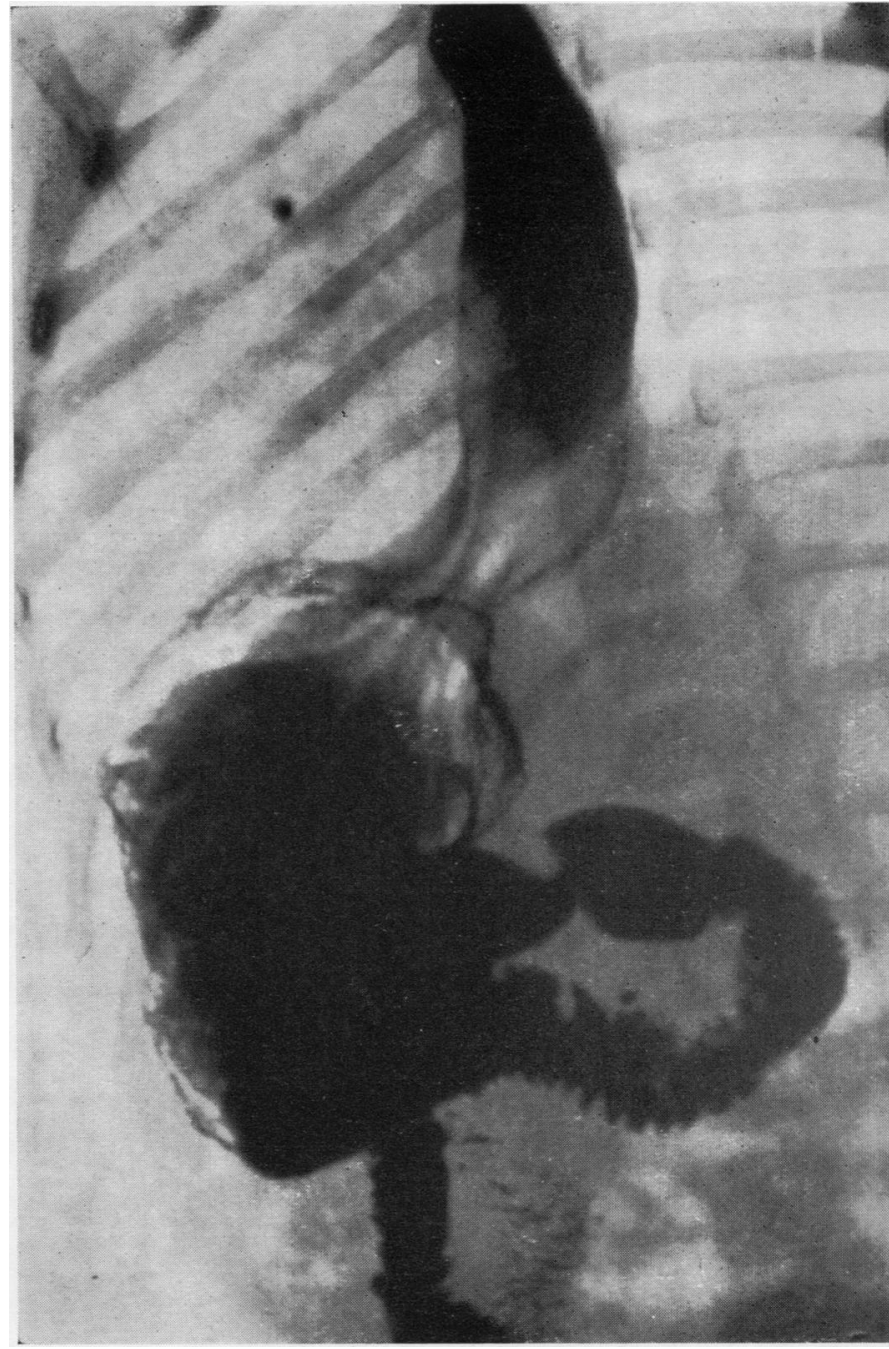

FIG. 3. regurgitation is sometimes clearly illustrated, and screening shows how the opaque medium in the oesophagus, hernia, and stomach follows the movements of the table.

Second Type of Short Oesophagus.-The second variety (Fig. 3) of short oesophagus was demonstrated in six children. As a rule the oesophagus is dilated, and, unlike the first variety, there is no constriction at a level with the cardia. Since the hernia is of the same width as the oesophagus or only slightly wider, radiographs give the appearance that the broad oesophagus is of normal length, opening into the stomach below the diaphragm. Nevertheless, these cases also have a broad hiatus oesophagus and the fundus is characteristically funnel-shaped. The radiographs, moreover, show continuation of the gastric mucosal relief through the hiatus into the hernia. Lastly, the children often exhibit rather massive regurgitation of contrast medium from the stomach into the oesophagus when lying in the horizontal or the Trendelenburg position. On the basis of these findings, a diagnosis of short oesophagus was made in six cases and verified by surgery in five. The patients were 
2 months, 18 months, 18 months, 21 months, 3 years, and $4 \frac{1}{2}$ years of age respectively. The symptoms found in this group did not differ from those in the former group. It was not possible to demonstrate a peptic ulcer. This group may be only a precursor of the first, and as yet we have been unable to produce proof, since we have not had the opportunity to follow the course of the disease in these children.

In 1947 Neuhauser and Berenberg described a syndrome in which the radiological changes closely resembled this second variety of short oesophagus. In 12 infants with persisting or recurrent vomiting they found a wide oesophagus, narrowing only slightly at the site of its passage through the hiatus. The cardial sphincter did not function at all, and in the horizontal position regurgitation of opaque medium was seen from the stomach into the oesophagus. Neuhauser and Berenberg interpreted the condition as a relaxation of the cardial sphincter due to a disturbance of the parasympathetic-sympathetic balance which regulates its function. They stated that they had had no opportunity to investigate the correctness of this theory at necropsy. They succeeded in controlling the symptoms by making the babies sit during and after meals and, in particularly pronounced cases, letting them assume a semi-sitting position day and night. At follow-up examination several months later even the radiological changes had subsided. If these cases are to be interpreted as short oesophagus, as indicated by the films, the treatment may have produced a spontaneous reduction of the hernia.

\section{Complications}

Short oesophagus is often complicated by oesophagitis or oesophagitis and peptic ulcer of the oesophagus due to the action of gastric acid upon the oesophageal mucosa. In oesophagitis the mucosal relief is irregular or indistinct.

In 12 cases of the former group oesophageal ulcer was diagnosed. In the latter group, radiographs failed to show signs of ulcer although four of the patients had haematemesis. The ulceration, appearing on the films in the form of a niche, is often at the lower end of the oesophagus, and only exceptionally higher up. In most cases it has its site on the posterior wall. The niche is usually small, about the size of a hempseed, but in one instance it was of some size, measuring $2 \frac{1}{2} \times \frac{1}{2} \mathrm{~cm}$. The ulceration nearly always entails constriction of the surrounding part of the oesophagus. In fresh cases or acute exacerbation of chronic ulcers, the constriction is due chiefly to oedema or inflammatory infiltration. In such there is pronounced stenosis (Fig. 4) and often considerable dilatation of the upper part of the oesophagus. At this stage it is not always possible to demonstrate the ulceration, but if dietetic management is carried on for some time the stenosis and dilatation subside and the niche is sometimes demonstrable at the site of the constriction.

In chronic or healed ulcers there is also a narrowing of the oesophagus due mostly to cicatricial changes in the mucosa. The constricted area is usually longer and the stenosis and dilatation not as marked. Sometimes an ulcer niche is demonstrable, most frequently at the lower end of the constriction (Fig. 5).

\section{Radiological Findings}

As will be seen from the table setting out the series, some of the children have been in hospital repeatedly and 11 have been followed up. This has given us the opportunity of observing the radiological changes for a number of years. In early 
FIG. 4.-Marked dilatation of the upper end of the oesophagus in a case of ulcer.
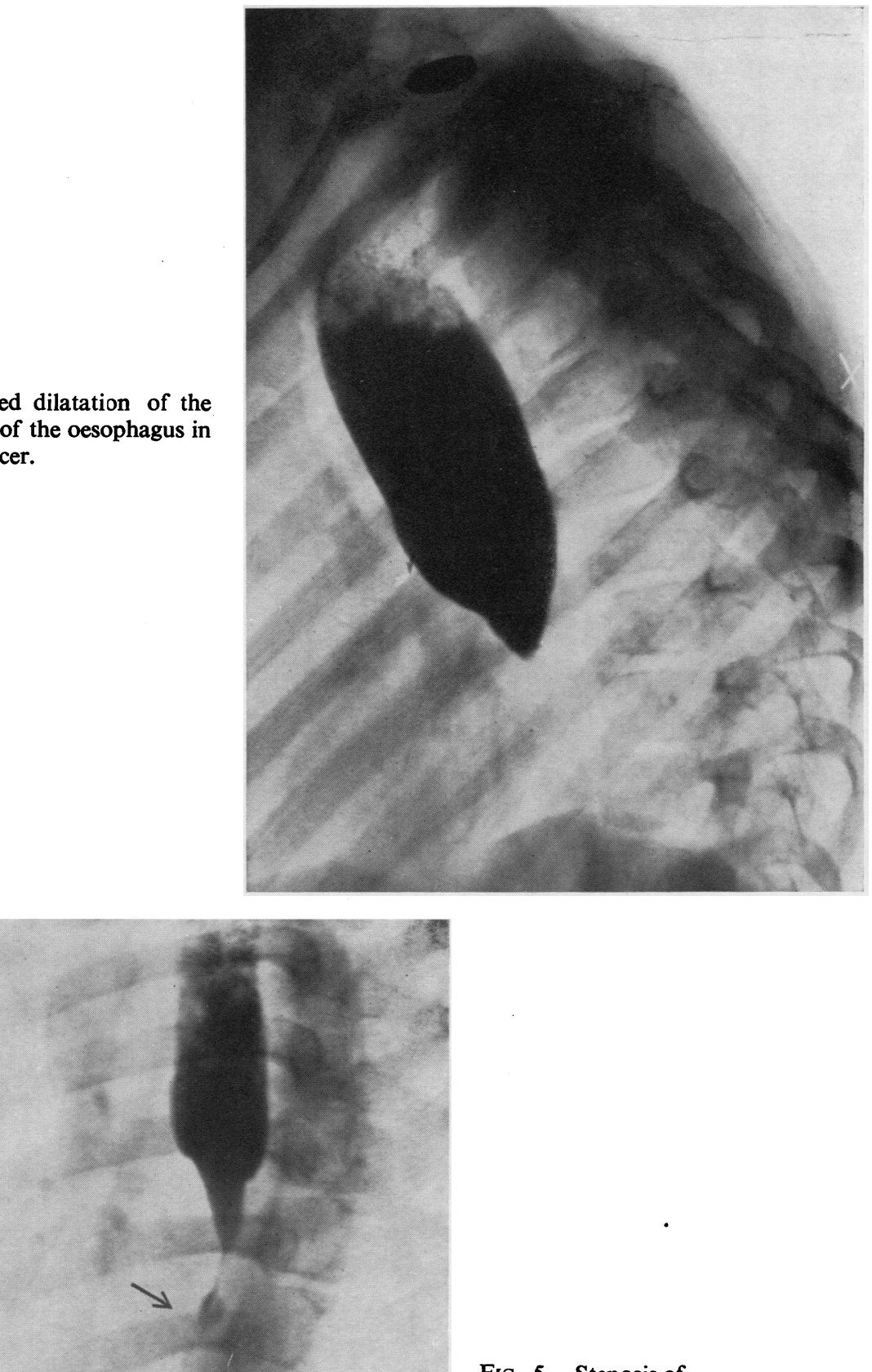

Fig. 5.-Stenosis of the oesophagus (arrow) in a case of chronic ulcer. 
childhood there is usually only slight dilatation of the oesophagus and the constricted area at the cardial end is rather short. Secondary changes are rare. It is not until the age of 3 or 4 that radiological signs of peptic ulcer appear. A niche was demonstrable in one child under 3 years of age, a girl. As the ulceration appears, the corresponding part of the oesophagus becomes constricted and the overlying area dilated. If the ulcer heals or becomes chronic, cicatricial changes ensue. The periodical exacerbations, so characteristic of the disease, are often due to an acute flare-up of a chronic, or the formation of a new, ulcer. In a few cases this gives rise to further cicatricial changes which lengthen the stenosis. In others the constricted area remains almost unchanged. The hernia gradually increases in size, due more to the normal alteration in the shape of the thorax and flattening of the diaphragm than to further shortening of the oesophagus.

The technical procedure of the radiological examination did not differ essentially from that used ordinarily in the examination of the oesophagus and stomach except that particular importance was attached to demonstrating regurgitation. In some cases the children cried so much in the course of the examination that the resulting excess pressure in the chest prevented regurgitation, even in the Trendelenburg position. As soon as they could be calmed down, reflux of opaque medium into the oesophagus was seen. Other children refused to take the contrast meal in the $x$-ray department, so it was impossible to observe the passage of the barium meal down the oesophagus, but by administering the barium in the ward before the examination the presence of short oesophagus was demonstrable by regurgitation.

The radiological changes in the oesophagus and stomach in patients suffering from short oesophagus are not apt to be mistaken for other morbid conditions in these organs. On the other hand, it may be difficult to demonstrate their presence if they are slight. When symptoms indicate the presence of short oesophagus it is important to repeat the examinations until the diagnosis can be confirmed or ruled out with certainty.

\section{OESOPHAGOSCOPY}

In addition to radiological examination, oesophagoscopy must be used to diagnose the condition, but, since this procedure was carried out in only a few of the cases, our experience of the method is too limited to allow of an estimation of its value.

\section{Prognosis}

Conservative Treatment.-The disease is seldom serious, but unpleasant for the child as well as its parents, and the chances of cure by conservative treatment are slight. This may be illustrated by a follow-up examination in April, 1949, of the cases not operated upon known at that time, a total of 14 patients of whom one died and two failed to reply to the inquiry. This leaves 11 patients, who were re-examined and showed the following symptoms.

\section{Present Symptoms}

\begin{tabular}{|c|c|c|c|c|c|c|}
\hline Vomiting & .. & .. & .. & $\cdots$ & $\ldots$ & in 11 cases \\
\hline 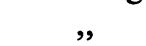 & and pain ... & $\cdots$ & $\cdots$ & $\cdots$ & . . & , $5 \quad$ \\
\hline ," & „, dysphagia & .. & .. & . & .. &, 5 \\
\hline , & ". haemorrhage & & . & . & . & "1 case \\
\hline , & pain, and haemor & rrhages & & .. & .. & , $1 \quad$, \\
\hline
\end{tabular}


Age Distribution

\begin{tabular}{|c|c|c|c|c|c|c|c|}
\hline $0-1$ year & $\cdots$ & $\cdots$ & $\cdots$ & $\cdots$ & - & . & 1 patient \\
\hline $2-6$ years & .. & $\cdots$ & .. & . & . & , & 2 patients \\
\hline $6-10$, & . & . & . & . & . & .. & $2 \quad$ \\
\hline $12-14$, & .. & . & . & . . & . & . & 3 \\
\hline 20 & . & .. & . & . & . & $\ldots$ & 2 \\
\hline 31, & . & . & . & . & . & . & 1 patient \\
\hline
\end{tabular}

Condition at Follow-up Examination

\begin{tabular}{|c|c|c|c|c|c|}
\hline Unchanged .. & - & .. & . & .. & 7 patients \\
\hline Slightly improved .. & $\cdots$ & .. & . & .. & 2 \\
\hline Considerably improved & . & . & & .. & 2 \\
\hline Symptomless .. .. & .. & $\ldots$ & $\ldots$ & $\ldots$ & None \\
\hline
\end{tabular}

According to these data, the chances of cure from conservative treatment are poor, only two of the patients feel better, whereas in nine the condition is unchanged or only slightly improved and none has obtained complete relief. Children afflicted with this disease are bound to suffer from vomiting for years even though they may be symptomless for some length of time in between. The purely symptomatic conservative treatment of overcoming the bad spells by giving predominantly fluids in small portions swallowed with ample water does not alter the prognosis. Some of the patients have benefited by a simultaneous medication with phenobarbital, and a few have remained symptomless for some time after a course of daily bouginage. It is beyond doubt that operation is called for in all severe cases of short oesophagus. It is only in mild cases that it is justified to observe the patient for a few weeks, noting the general condition, the frequency and intensity of the vomiting, and the possible attendant haematemeses.

Surgical Treatment.-The proof that the condition cannot be interpreted as a congenital short oesophagus, but as a congenital hernia-a sliding hernia-in which the stomach and cardia force their way into the chest making the oesophagus retract, lies in operation before severe secondary changes have occurred in the form of fibroid shrinkage of the oesophageal wall and fixing of the oesophagus to the surrounding tissues due to peri-oesophagitis. In our experience, radiological examination cannot decide whether it will be possible to stretch the oesophagus so far as to get the cardia below the diaphragm. The route should always be transthoracic, since it may prove necessary to dissect and detach the oesophagus as far as the aorta. The operation we have used consists in freeing the stomach and oesophagus so that the stomach and the cardia may be fixed below the diaphragm, the hiatus repaired, and the cardial closing mechanism re-established. In five cases this has proved very easy; the stomach and oesophagus could be mobilized without the slightest difficulty and the cardia brought down below the diaphragm without any resistance from the soft and elastic oesophagus. In one case the mediastinal connective tissue has been hyperaemic and oedematous, and there have been numerous large hyperplastic lymph nodes, particularly around the cardia. The dissection and detachment of the oesophagus have had to be more careful, involving ligation of blood vessels. But when this has been done it has been easy to place the cardia below the diaphragm and to close the hiatus around the oesophagus. Lastly, in 
two cases the mediastinal connective tissue has been the seat of widespread fibroid changes fixing the oesophagus to the surrounding tissues. The oesophageal wall proper has undergone fibroid changes and the lumen narrowed. Moreover, there are cases of large peptic ulcers, and we have encountered two. In one the ulcer had encroached upon the aortic wall and had to be removed by sharp dissection together with a piece of the adventitia of the vessel.

It may be difficult to decide whether the oesophageal constriction is due to spasm or fibroid changes. In one instance it was due essentially to pressure from lymph nodes and peri-oesophagitic strands. In these cases it has been necessary to free the stomach and the oesophagus from the diaphragm as far as the site where the oesophagus disappears behind the aorta. The oesophagus has had to be dissected carefully from the inferior left pulmonary vein and the left main bronchus. It is important to detach the oesophagus as far as the aorta and pull the oesophagus far forward in the hiatus, thus reaching the summit of the dome of the diaphragm. In that way $3 \mathrm{~cm}$. can be gained.

After the detachment it has been possible to pull the cardia down in all cases but two. In one of them it would no doubt have been possible with our present experience: in the other oesophago-gastrectomy was required because of a penetrating peptic ulcer.

Operative Technique.-The anaesthetic was intratracheal nitrous oxide-oxygen ether. In no case did tracheotomy become necessary because of glottic oedema.

A postero-lateral incision was made on the left side, resecting the eighth rib. After dividing the mediastinal pleura and detaching the stomach and oesophagus, the hernial sac was opened, the peritoneum attaching to the stomach being cut through. Thereupon the diaphragmatic crura are dissected, revealing the red bundles of muscle, especially posteriorly, where they converge into a V-shaped figure. A silk suture is passed through the crura anteriorly in the hiatus, including the oesophageal wall, after which the hiatus is closed from behind with three to four silk sutures, the last of which includes the oesophageal wall. Moreover,' a few silk sutures are passed through the crura and the oesophageal wall.

As a safety measure, a tube may be passed into the stomach and left in place during the process of suturing. The chest wall is closed with silk sutures and a Pezzer catheter inserted into the chest and left there for 24 hours.

Results of Surgical Treatment.-One patient had a penetrating ulcer and required oesophago-gastrectomy. Now, he really has a too-short oesophagus affording a possibility of regurgitation, and the prognosis is doubtful, although vagotomy has been performed.

One patient underwent operation, but at that time it was not considered possible to place the cardia below the diaphragm. This patient is still suffering from regurgitation and haematemeses and is to be admitted for another operation.

In eight cases herniotomy was performed on the hiatus and the cardia was fixed below the diaphragm.

Follow-up examinations from 15 to 2 months after the operation showed that seven patients were in excellent health without symptoms, whereas one had a recurrence and probably will need another operation. 


\section{ConcLusion}

The numbers involved are small and the follow-up period short, but according to our experience a congenital short oesophagus does not exist or is extremely rare. These patients are suffering from a congenital hiatal hernia. Before operation it is impossible to know whether the cardia can be brought below the diaphragm. It is difficult to decide whether a narrowing of the oesophagus is due to spasm or stricture. The approach must be through the thorax.

In view of improved anaesthetic technique and thoracic operations in early childhood and the low mortality which they carry, all cases of so-called " congenital short oesophagus" should be submitted to operation, if possible before secondary changes, such as shrinkage and fixation of the oesophagus, and peptic ulcer are caused by the regurgitation. The operation is then a simple procedure which relieves the patient of an extremely unpleasant and in some cases fatal disease.

\section{SUMMARY}

"Brachyoesophagus," or congenital short oesophagus as it has been called, is now interpreted as a congenital hernia through the hiatus oesophagus with simultaneous shortening of the oesophagus. The anomaly was first described in 1918, and from 1930 it has been diagnosed in ever-increasing numbers, the number of reported cases being about 100 . We have diagnosed 24 cases, 14 of which were recognized after 1947.

According to Allison (1948), the primary factor in adults is a hiatal hernia, and regurgitation of gastric contents produces oesophagitis and perhaps peptic ulcer of the oesophagus. Cicatricial changes contribute to shortening and fixing the oesophagus.

As a rule the symptoms set in immediately after birth, most commonly in the form of vomiting, nearly always periodical. Further symptoms are haemorrhage, either in the form of a slightly blood-tinged vomitus or of actual haematemesis, loss of weight, dysphagia, pain, anaemia, and in some cases dehydration.

The differential diagnostic possibilities are: hyper- or hypo-galactia, congenital pyloric stenosis, atresia of the oesophagus and duodenal stenosis, and in older children gastric lesions, especially gastric or duodenal ulcer.

The diagnosis is confirmed by radiological examination after administering an opaque medium. It is important to examine the patient in the Trendelenburg position to demonstrate regurgitation. By this procedure it is possible to demonstrate two types of lesion. (1) A short and somewhat dilated oesophagus, opening directly into the hiatal hernia with a constriction at the site of transition. This is the most common variety and probably a further development of Type 2. (2) A dilated oesophagus without constrictions. The hernia is of the same width as the oesophagus, which appears to be of normal length and to open into the stomach below the diaphragm. The possibility of spontaneous reduction may be demonstrated.

The condition is often complicated by "oesophagitis" or by peptic ulcer of the oesophagus, more rarely by perforation. In most cases the disease runs a prolonged course, characterized by intermittent, ever-recurring vomiting.

In 11 patients who had been treated conservatively, the condition had remained completely unchanged in seven. Two showed slight and two marked improvement, but none was symptomless, and all were still troubled by vomiting. 
Conservative medical management must be abandoned, and early operation recommended. At operation, the stomach and oesophagus are freed, so that the stomach and cardia may be fixed below the diaphragm. At the same time herniotomy is performed, and, after isolation of the diaphragmatic crura, sphincter function is re-established by sutures through the crura and the oesophageal wall.

Follow-up examination of 10 operated cases from 15 to 2 months after the operation revealed that seven patients were symptom-free and only one had recurrence. In the remaining two cases the operation was technically impracticable.

\section{REFERENCES}

Ákerlund, Åke (1926). Acta radiol., Stockh., 6, 3.

Allison, P. R. (1948). Thorax, 3, 20.

Neuhauser, E. B. D., and Berenberg, W. (1947). Radiology, 48, 480.

Olsen, A. M., and Harrington, S. W. (1948). J. thorac. Surg., 17, 189.

Thomsen, G. (1949). Acta radiol., Stockh., 32, 193.

Wamberg, E. (1947). Acta paediatr., Stockh., 34, 293. 